\title{
AGING AND THERAPEUTIC DELAY IN COLORECTAL CANCER: A FRENCH POPULATION-BASED STUDY
}

\author{
C. Montuclard ${ }^{1}$, V. Jooste', V. Quipourt ${ }^{2}$, S. Marilier', J. Faivre ${ }^{1}$, C. Lepage ${ }^{1}$, A.M. Bouvier ${ }^{1}$
}

\begin{abstract}
Background/Objectives: Data on the time between colorectal cancer diagnosis and treatment in real-life practice for elderly patients are scarce. We measured times from diagnosis to first-course therapy in elderly patients with colon and rectal cancers. Design: The study was carried out on the population-based Burgundy Digestive Cancer Registry (France). Setting: Therapeutic delays were described by medians and interquartile ranges and compared by the Kruskal-Wallis rank test. Factors associated with changes in therapeutic delay were identified using a multivariate Cox model. Participants: The analysis was carried out on 2,884 patients aged 60 years and over with colorectal adenocarcinoma diagnosed between 2005 and 2011. Measurements and Results: The median therapeutic delay for colon cancer was 25 days in patients aged 60 to 69 years and 24 days for those aged $70-79$ years. The delay fell significantly to 19 days in patients aged 80 and over $(p<0.001)$. The median therapeutic delay for rectal cancer did not vary according to age group (respectively 39, 38 and 33 days). For colon cancer, a Charlson comorbidity score $=0$, in all age groups, and private care for patients under 80 years, significantly shortened the therapeutic delay. It was significantly longer during the period [2008-2011] only in patients under 80 (HR: 0.89 [0.81 - 0.99] p=0.037). For rectal cancer, only advanced stage (HR advanced vs II: 1.39 [1.04-1.86], $\mathrm{p}=0.025)$ shortened the therapeutic delay in patients under 80 , while private care shortened therapeutic delay only in patients over 80 (HR private vs public: 1.66 [1.00-2.74], $\mathrm{p}=0.049$ ). Conclusion: This study highlights that differences in therapeutic delay for the elderly increased over time for colon and rectal cancer. The therapeutic delay did not differ much between the 60-69 and the 70-79 years age groups, whereas it was shorter for patients aged 80 and over.
\end{abstract}

Key words: Colorectal cancer, elderly, quality of life, registry, adjuvant treatments.

Abbreviations: HR: hazard ratio, CI: confidence interval.

\section{Introduction}

In France, colorectal cancer is the third most common cancer with an annual incidence of 40,000 (1) new cases. The ageing of the population and the rise in life expectancy, as well as the increasing incidence of colorectal cancer, will lead to a growing number of affected patients. Social inequalities in healthcare have been identified in every industrialized country whatever the amount of money the government spends on the organization of the health system. In the second and third 'Cancer Plans' launched in France, the French authorities established as a national priority the reduction of inequalities with regard to the management of cancers. Reasons for disparities in the management of

1. Registre Bourguignon des Cancers Digestifs, Inserm U866 and CIC-EC, Dijon ; Université de Bourgogne; CHU Dijon; 2. Unité de Coordination en Oncogériatrie en Bourgogne (UCOGB), Hôpital de jour gériatrique, Centre de Champmaillot, CHU Dijon.

Corresponding Author: Anne-Marie Bouvier, Registre Bourguignon des Cancers Digestifs [INSERM U866], Faculté de Médecine, BP 8790021079 Dijon Cedex, France. Tel : (+33) 380393340 Fax : (+33) 3806682 51, E-mail : anne-marie. bouvier@u-bourgogne.fr cancer are multiple and complex. The age of patients is an established factor associated with disparities in the management of colorectal cancer (2). A number of mechanisms lie at the root of these disparities and can intervene at every step of the management, from the pre-diagnostic to the post-therapeutic phase. One of the factors that may determine the care of patients and the prognosis of the disease is the therapeutic delay. Recommendations concerning the therapeutic delay are scarce in Europe. Only the United Kingdom and Denmark produced guidelines recommending a limited delay between diagnosis and treatment. The precise measurement of the disparities that can exist between various groups of patients concerning the therapeutic delay requires data representative of the general population. In this context, cancer registries are a source of extremely useful data. The aim of this study was to describe the therapeutic delay in patients with colorectal cancer according to their age, and to identify epidemiological characteristics associated with disparities in this delay using a population-based digestive cancer registry. 
Table 1

Characteristics of the population according to location of the cancer

\begin{tabular}{|c|c|c|c|c|c|c|c|c|c|c|}
\hline & & & Colon & & & & & Rectum & & \\
\hline & & $\begin{array}{l}60-69 \\
\text { years }\end{array}$ & $\begin{array}{l}70-79 \\
\text { years }\end{array}$ & $\begin{array}{c}\geq 80 \\
\text { years }\end{array}$ & & & $\begin{array}{l}60-69 \\
\text { years }\end{array}$ & $\begin{array}{l}70-79 \\
\text { years }\end{array}$ & $\geq 80$ years & \\
\hline & $\mathbf{N}$ & $\%$ & $\%$ & $\%$ & $\mathrm{p}$ & $\mathbf{N}$ & $\%$ & $\%$ & $\%$ & $\mathrm{p}$ \\
\hline Sex & & & & & $<0.001$ & & & & & 0.035 \\
\hline Male & 1228 & 61 & 58 & 45 & & 381 & 66 & 65 & 54 & \\
\hline Female & 1041 & 39 & 42 & 55 & & 234 & 34 & 35 & 46 & \\
\hline Place of residence & & & & & 0.452 & & & & & 0.945 \\
\hline Urban & 1423 & 61 & 62 & 64 & & 360 & 58 & 59 & 58 & \\
\hline Rural & 846 & 39 & 38 & 36 & & 255 & 42 & 41 & 42 & \\
\hline Charlson comorbidity score* & & & & & $<0.001$ & & & & & $<0.001$ \\
\hline 0 & 1087 & 59 & 45 & 45 & & 344 & 72 & 52 & 45 & \\
\hline 1 & 581 & 21 & 28 & 28 & & 141 & 16 & 24 & 30 & \\
\hline$>1$ & 564 & 20 & 27 & 28 & & 122 & 11 & 24 & 25 & \\
\hline Period at diagnosis & & & & & 0.025 & & & & & 0.038 \\
\hline $2005-2007$ & 985 & 44 & 46 & 44 & & 264 & 36 & 45 & 49 & \\
\hline $2008-2011$ & 1284 & 56 & 54 & 56 & & 351 & 64 & 55 & 51 & \\
\hline Health care facility & & & & & $<0.001$ & & & & & 0.179 \\
\hline Public & 911 & 25 & 38 & 53 & & 95 & 14 & 15 & 19 & \\
\hline Private & 898 & 48 & 39 & 34 & & 262 & 49 & 41 & 37 & \\
\hline Mixed & 460 & 27 & 23 & 13 & & 258 & 37 & 44 & 44 & \\
\hline First treatment & & & & & 0.012 & & & & & 0.009 \\
\hline Surgery & 2129 & 92 & 93 & 96 & & 229 & 31 & 36 & 46 & \\
\hline $\mathrm{CT}$ and / or RT & 140 & 8 & 7 & 4 & & 386 & 69 & 64 & 54 & \\
\hline TNM stage ${ }^{* *}$ & & & & & $<0.001$ & & & & & 0.224 \\
\hline I & 429 & 23 & 21 & 14 & & 192 & 37 & 32 & 24 & \\
\hline II & 831 & 30 & 38 & 40 & & 133 & 20 & 20 & 26 & \\
\hline III & 508 & 23 & 21 & 24 & & 120 & 17 & 21 & 20 & \\
\hline Advanced \# & 494 & 24 & 20 & 22 & & 168 & 26 & 27 & 30 & \\
\hline
\end{tabular}

Unknown: *45 cases, ** 9 cases. CT: chemotherapy, RT: radiotherapy, \# Including patients with visceral metastasis and non-resected tumours without evidence of visceral metastasis

\section{Materials and methods}

\section{Study population}

A population-based cancer registry records all digestive cancers in two French administrative areas in Burgundy: Côte-d'Or and Saone-et-Loire (1,064,000 inhabitants according to the 2011 census). Information is regularly obtained from pathologists in public and private practice, public hospitals (university hospitals including the cancer center and general hospitals), and private physicians: gastroenterologists, surgeons, oncologists, radiotherapists, general practitioners, hospital administrative databases, and the National Health System database and death certificates. No case is recorded through death certificates alone, but these are used as an identification source. Because of the multiplicity of medical and administrative information sources, it was assumed that nearly all newly diagnosed cancers had been registered. The quality of the data collection is evaluated every 4 years by the Institut National de la Santé et de la Recherche Médicale (INSERM), the Institut de Veille Sanitaire (InVS) and the Institut National du Cancer (INCa).Cancers were classified according to the International Classification of Diseases, 10th revision (3). Therapeutic delay was defined as the time between date of diagnosis and first oncologic treatment performed (surgery, radiotherapy or chemotherapy).

Overall 3,679 invasive colorectal adenocarcinomas were registered between 2005 and 2011 in patients aged 60 years and over. In case of two synchronous colorectal 
Table 2

Therapeutic delay (days) for colon cancer by age group

\begin{tabular}{|c|c|c|c|c|c|c|c|c|c|c|}
\hline Male & 356 & 26 & [14-42] & 512 & 25 & [14-40] & 360 & 19 & [9-35] & $<0.001$ \\
\hline Female & 229 & 24 & [15-35] & 374 & 24 & [12-38] & 438 & 18 & [8-31] & $<0.001$ \\
\hline Urban & 359 & 24 & [14-38] & 550 & 24 & [13-39] & 514 & 20 & [9-32] & $<0.001$ \\
\hline Rural & 226 & 26 & [14-42] & 336 & 26 & [13-41] & 284 & 15 & [8-34] & $<0.001$ \\
\hline \multicolumn{11}{|l|}{ TNM stage } \\
\hline $\mathrm{I}$ & 131 & 36 & [25-52] & 182 & 37 & [21-54] & 116 & 30 & [18-48] & 0.019 \\
\hline \multicolumn{11}{|c|}{ Period at diagnosis } \\
\hline 2005-2007 & 256 & 23 & [13-37] & 411 & 23 & [13-37] & 318 & 18 & [8-32] & $<0.001$ \\
\hline 2008-2011 & 329 & 27 & [15-40] & 475 & 26 & [14-41] & 480 & 19 & [9-32] & $<0.001$ \\
\hline \multicolumn{11}{|c|}{ Charlson comorbidity score } \\
\hline 0 & 342 & 25 & [14-39] & 396 & 23 & [13-37] & 349 & 18 & [9-29] & $<0.001$ \\
\hline 1 & 120 & 24 & [15-34] & 244 & 25 & [14-40] & 217 & 19 & {$[9-34]$} & 0.002 \\
\hline$>1$ & 114 & 30 & {$[15-43]$} & 235 & 26 & {$[12-42]$} & 215 & 19 & {$[8-36]$} & 0.002 \\
\hline \multicolumn{11}{|c|}{ Health care facility } \\
\hline
\end{tabular}

IQ: inter quartile interval, \# Including patients with visceral metastasis and non-resected tumours without evidence of visceral metastasis

cancers, the shortest therapeutic delay was taken into account for the analyses. To avoid including patients with an irrelevant therapeutic delay, we excluded patients with synchronous non-colorectal cancer, patients who underwent emergency surgery (obstruction or perforation), patients treated by endoscopic resection during the diagnostic colonoscopy, patients with fortuitous diagnosis during a surgery for another reason, and patients not treated. A total of 774 patients were excluded. The therapeutic delay was unknown for 21 patients. Finally, 2,884 patients were included (79\% cases with colon cancer and $21 \%$ with rectal cancer).

\section{Data collection}

The Digestive cancer registry contains key information routinely collected regarding patients' characteristics (sex, age, place of residence, comorbidities, health care facilities), the tumor (stage at diagnosis), and treatment (date and nature of treatment including surgery, chemotherapy and radiotherapy). Patients were divided into three age groups: age 60 to 69,70 to 79 and 80 and above. The period of diagnosis was divided into two groups, [2005-2007] and [2008-2011]. Cancer stage at the time of diagnosis was classified according to the TNM classification (4). Advanced cancer was defined by cancer stage IV and non-metastatic/ non-resected cancer. The place of residence was categorized according to the classification established by the French National Statistics and Economic Studies Institute (INSEE) into urban and rural areas. The date of diagnosis was generally defined by the date of the colonoscopy. When a colonoscopy had not been performed before treatment, the date of diagnosis was the date of the first positive imaging (CT-scan or barium enema). Comorbidities at the time of diagnosis were listed using the Charlson index (5). Individuals were then assigned to a group according to their index: 0,1 or $>1$. The healthcare delivery pattern was classified into public hospitals (including university 
Table 3

Factors associated with quicker access to treatment after diagnosis of colon cancer according to age (Cox proportional hazards model)

\begin{tabular}{|c|c|c|c|c|c|c|}
\hline \multirow{2}{*}{ Sex } & \multicolumn{3}{|c|}{$<80$ years } & \multicolumn{3}{|c|}{$\geq 80$ years } \\
\hline & HR & {$[95 \% \mathrm{CI}]$} & $\mathbf{p}$ & HR & {$[95 \% \mathrm{CI}]$} & $\mathrm{p}$ \\
\hline Male & 1 & & & 1 & & \\
\hline Female & 1.02 & [0.92 - 1.14] & 0.658 & 1.08 & [0.93 - 1.26] & 0.290 \\
\hline Rural & 0.98 & [0.88 - 1.09] & 0.722 & 1.07 & [0.93 - 1.25] & 0.340 \\
\hline \multicolumn{7}{|l|}{ TNM stage } \\
\hline I & 0.58 & [0.50 - 0.67] & $<0.001$ & 0.55 & [0.44 - 0.69] & $<0.001$ \\
\hline II & 1 & & & 1 & & \\
\hline \multicolumn{7}{|c|}{ Period at diagnosis } \\
\hline 2005-2007 & 1 & & & 1 & & \\
\hline $2008-2011$ & 0.89 & [0.81 - 0.99] & 0.037 & 0.94 & [0.81 - 1.09] & 0.377 \\
\hline \multicolumn{7}{|c|}{$\begin{array}{l}\text { Charlson comorbi- } \\
\text { dity score \# }\end{array}$} \\
\hline 0 & 1 & & & 1 & & \\
\hline 1 & 0.95 & [0.84 - 1.09] & 0.492 & 0.84 & [0.70 - 0.99] & 0.046 \\
\hline$>1$ & 0.84 & [0.74-0.96] & 0.013 & 0.89 & [0.74 - 1.07] & 0.208 \\
\hline \multicolumn{7}{|c|}{ Health care facility } \\
\hline
\end{tabular}

HR>1means quicker access to treatment, \# Including patients with visceral metastasis and non-resected tumours without evidence of visceral metastasis

hospitals, general hospitals and cancer therapy centers), private hospital and mixed places of treatment (receiving care in both public and private hospitals).

\section{Statistical analysis}

The characteristics of the cohort were described using percentages. The therapeutic delay was calculated in days and was described by medians and inter quartile intervals (IQI). Medians were compared between different groups by Kruskal-Wallis tests in univariate analysis. Multivariate Cox proportional hazards models were used separately for colon and rectal cancer cases in order to determine what factors were associated with a greater probability of quick access to treatment. As radiotherapy or chemotherapy were very rare as first treatment for colon cancer, this variable was only analyzed for rectal cancer.

\section{Results}

Table 1 shows the descriptive data for the whole population according to cancer location. The distributions of the main variables did not differ by age group between colon and rectal location. For both colon and rectal cancers, there was a greater proportion of women in patients over 80 than in younger patients. In patients over 80 with colon cancer, the proportion of patients managed in public facilities was higher than the proportion of patients managed in private facilities (53\% vs. 34\%) whereas patients under 80 were more often managed in private facilities. Colon cancer was diagnosed in a context of sub-occlusion for 5\% and 6\% of patients aged 60 to 69 and 70-79, respectively, and for $9 \%$ of those over 80 .

For rectal cancer, the proportion of patients receiving chemo or radiotherapy at first treatment was lower in the oldest age group than in the youngest ( $54 \%$ vs. $69 \%$ ). Overall, $45 \%$ of patients aged over 80 had a Charlson 
Table 4

Therapeutic delay (days) for rectal cancer by age group

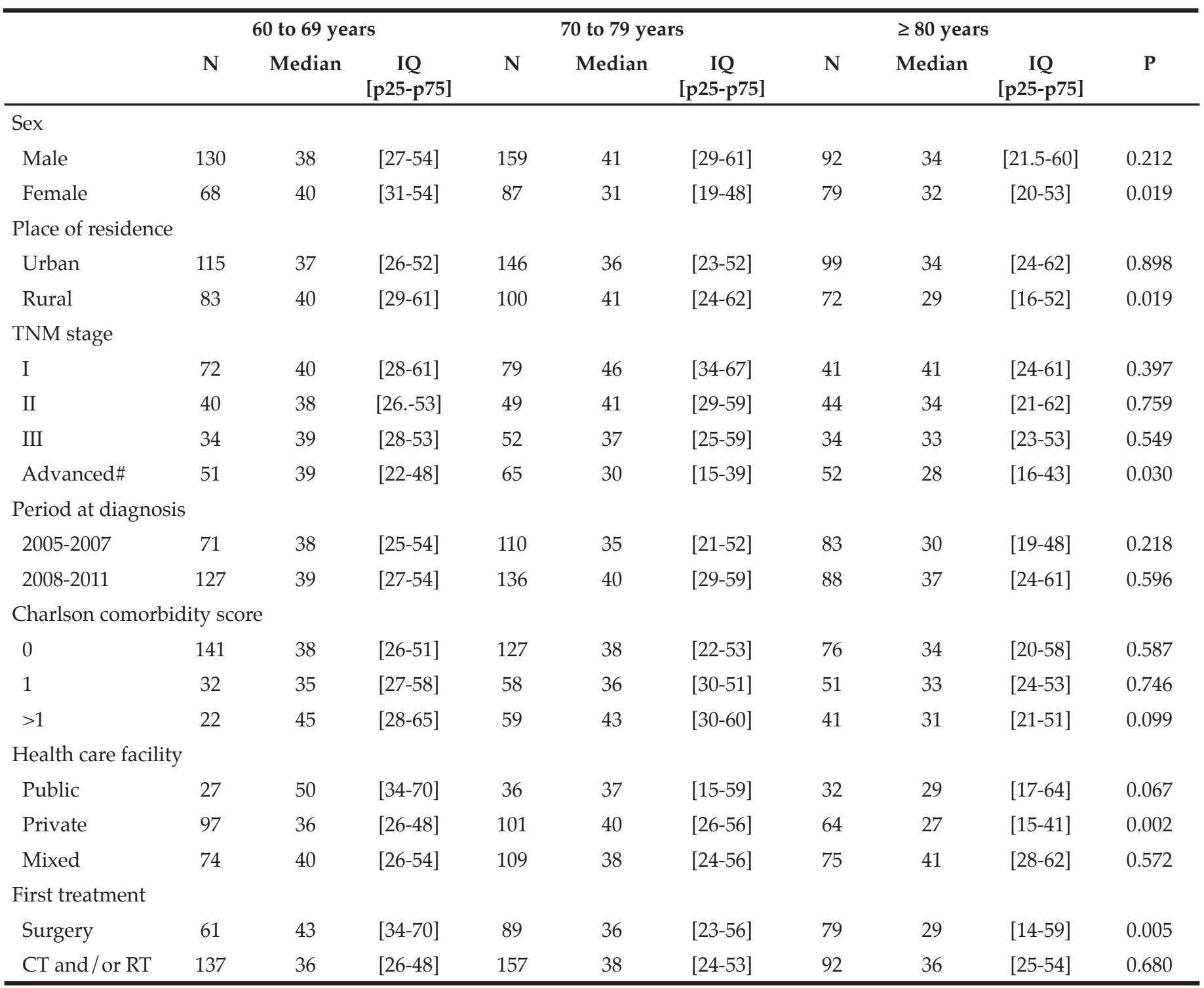

IQ: inter quartile interval, CT: chemotherapy, RT: radiotherapy, \#Including patients with visceral metastasis and non-resected tumours without evidence of visceral metastasis

score $=0$. This proportion for the $60-69$ year-olds was $59 \%$ for colon cancer and $72 \%$ for rectal cancer.

The median delays between diagnosis and treatment and their variations according to age groups and patients' characteristics for colon and rectal cancers were assessed. The median therapeutic delay was 25 days 95\% CI [13-41] for the whole population. Delays were shorter for colon cancer (22 days 95\% CI [12-36]) than for rectal cancer (37 days $95 \% \mathrm{CI}[24-55])$.

\section{Colon cancer}

The overall median therapeutic delay for colon cancer was 25 days $95 \%$ CI [14-39] in patients aged 60 to 69 years and 24 days $95 \% \mathrm{CI}$ [13-39] for those aged 70-79 years. It decreased to 19 days $95 \% \mathrm{CI}$ [8-32] in patients aged 80 and over $(p<0.001)$. Therapeutic delays were significantly shorter in patients over 80 years compared with those aged less than 79 years for all studied variables except for patients managed in public and private facilities (Table 2). Neither sex nor place of residence was significantly associated with shorter access to treatment in the multivariate Cox analysis (Table 3). A Charlson comorbidity score of zero was significantly associated with a shorter therapeutic delay in patients in all age groups. Care in a private facility was associated with a shorter therapeutic delay only in patients under 80 (HR: 1.16, [1.03-1.31], $\mathrm{p}=0.017)$. The delay was significantly longer during the most recent time period only in patients under 80 (HR: 0.89, [0.81-0.99], $\mathrm{p}=0.037$ ) and for 
Table 5

Factors associated with quicker access to treatment after diagnosis of rectal cancer according to age

(Cox proportional hazards model)

\begin{tabular}{|c|c|c|c|c|c|c|}
\hline & & $<80$ years & & & $>80$ years & \\
\hline & HR & {$[95 \% \mathrm{CI}]$} & $\mathrm{p}$ & HR & {$[95 \% \mathrm{CI}]$} & $\mathrm{p}$ \\
\hline \multicolumn{7}{|l|}{ Sex } \\
\hline Male & 1 & & & 1 & & \\
\hline Female & 1.16 & [0.94- 1.42] & 0.165 & 1.12 & {$[0.80-1.56]$} & 0.521 \\
\hline \multicolumn{7}{|l|}{ Place of residence } \\
\hline Urban & 1 & & & 1 & & \\
\hline Rural & 0,9 & {$[0.74-1.10]$} & 0.313 & 1.21 & {$[0.85-1.70]$} & 0.285 \\
\hline \multicolumn{7}{|l|}{ TNM stage } \\
\hline I & 0.88 & {$[0.67-1.15]$} & 0.357 & 0.97 & {$[0.61-1.53]$} & 0.885 \\
\hline II & 1 & & & 1 & & \\
\hline III & 0.99 & {$[0.73-1.35]$} & 0,949 & 1.13 & {$[0.70-1.85]$} & 0.612 \\
\hline Advanced* & 1.39 & {$[1.04-1.86]$} & 0.025 & 1.22 & {$[0.78-1.90]$} & 0.383 \\
\hline \multicolumn{7}{|c|}{ Period at diagnosis } \\
\hline $2005-2007$ & 1 & & & 1 & & \\
\hline 2008-2011 & 0.86 & {$[0.71-1.05]$} & 0.141 & 0.77 & {$[0.55-1.06]$} & 0.116 \\
\hline \multicolumn{7}{|c|}{ Charlson comorbidity score } \\
\hline 0 & 1 & & & 1 & & \\
\hline 1 & 1.06 & {$[0.82-1.36]$} & 0.655 & 1.30 & {$[0.88-1.92]$} & 0.195 \\
\hline$>1$ & 0.84 & {$[0.64-1.08]$} & 0.176 & 1.52 & {$[0.98-2.34]$} & 0.060 \\
\hline \multicolumn{7}{|c|}{ Health care delivery patterns } \\
\hline Public & 1 & & & 1 & & \\
\hline Private & 1.14 & {$[0.84-1.54]$} & 0.405 & 1.66 & {$[1.00-2.74]$} & 0.049 \\
\hline Mixed & 0.98 & [0.71 - 1.35] & 0.892 & 0.96 & {$[0.55-1.66]$} & 0.882 \\
\hline \multicolumn{7}{|l|}{ First treatment } \\
\hline $\mathrm{CT}$ and / or RT & 1 & & & 1 & & \\
\hline Surgery & 0.82 & {$[0.65-1.04]$} & 0.098 & 0.96 & {$[0.65-1.42]$} & 0.845 \\
\hline
\end{tabular}

CT: chemotherapy, RT: radiotherapy , \#Including patients with visceral metastasis and non-resected tumours without evidence of visceral metastasis. HR $>1$ means quicker access to treatment

stage I for patients in all age groups.

\section{Rectal cancer}

The overall median therapeutic delay for rectal cancer did not significantly vary by age group. It was 39 days [20-56] in patients aged 60 to 69 years, 38 days [23-56] for those aged 70-79 years and 33 days [20-55] for those aged $\geq 80$ years. Therapeutic delays were significantly shorter in patients over 80 years than in younger patients for patients with an advanced stage cancer, those living in rural areas, those managed in private health care facilities and those with surgery as the first treatment (Table 4). After adjustment for all concerned variables, place of residence and first surgical treatment were no longer associated with a shorter therapeutic delay for all patients. Private facilities (HR private vs public: 1.66 [1.00-2.74], $\mathrm{p}=0.049$ ) were significantly associated with a shorter therapeutic delay for patients over 80 years (Table $5)$.

\section{Discussion}

Our study examines real-life disparities in care related to delay between diagnosis and treatment in the elderly with colon and rectal cancers. The strength of this study is that it relies on a large population-based sample, allowing separate analyses for colon and rectal cancers. The therapeutic delay did not differ much between the 60-69 and the 70-79 years age groups, 
whereas it was shorter for patients aged 80 and over. The therapeutic delay increased with time period for patients younger than 80 years old with colon cancer whereas it did not vary for the oldest patients and for patients with rectal cancer. For colon cancer, sub-occlusion at diagnosis was almost twice as common in the oldest age group than in the youngest, which may partly explain the shorter therapeutic delay for the oldest patients. Patients managed in private facilities and presenting with advanced tumors were more likely to have a short therapeutic delay, and comorbidities did not strongly interact with this delay.

Colorectal cancer is frequently encountered in the elderly: 60 to $70 \%$ of cases occur in subjects aged 65 or older, and around $45 \%$ in those over the age of 75 (6). The improvement in life expectancy plus the increased size of the elderly population have led to a growing number of patients with the disease. Therefore, the care of elderly patients with colorectal cancer is a challenge. Measuring the inequality of treatment in patients with carcinoma is a major concern for the French health authorities, as underlined by successive Cancer Plans established in France since 2003. Disparities in the management of cancer in the elderly are multiple and complex; they can concern patients' geographical, social or economic environment, and specific approaches are needed to reduce them. No association has been clearly established between therapeutic delay and all-cause death in colon and rectal cancer (7). This meta-analysis by Ramos et al. failed to demonstrate any association between therapeutic delay and the colorectal cancer stage. Data from the SEER program suggested that the shortest delay $(<1$ week) was associated with a higher risk of all-cause death than was the case with longer delays in colon cancer, whereas it had no impact on colon and rectal cancer-specific death (8). Nevertheless, in addition to the evident psychosocial stress for patients awaiting treatment, therapeutic delay among elderly patients may be a valuable indicator of health care quality.

Unlike other countries, France has the theoretical advantage of a relatively homogeneous population, all of whom have the same national health insurance access to care throughout their lives. Nevertheless, the waiting time from diagnosis to treatment may vary among the elderly. The precise measurement of the disparities that can exist between various groups of patients concerning these delays requires the recording of representative data of the general population. Due to unavoidable selection bias, hospital-based data cannot be considered representative. Population-based registries allow the collection of exhaustive data from all sectors of care in well-defined geographical areas.

There are few studies concerning the therapeutic delay for colorectal cancer in the general population. In our study, the median therapeutic delay was 22 days for colon cancer and 37 days for rectal cancer. A Canadian population-based study showed shorter median therapeutic delays: 12 days for the treatment of colorectal cancer in 2005 (9). In a recent populationbased study in the USA, median treatment delays were 13 days for colon and 16 days for rectal cancer8. Other studies demonstrated longer treatment delays for rectal than for colon cancer. However, it is difficult to compare estimates of delay across studies due to differing definitions of delay (10). No previous study has been specifically dedicated to elderly patients and guidelines for maximum acceptable delays are still vague. For example, United Kingdom guidelines state that patients with cancer should have treatment initiated within 2 months of referral by general practitioners (11-13). In Denmark, the recommended maximum interval between referral and the work-up for colorectal cancer is 14 days, with the beginning of treatment within an additional 14 days (14). In France, there are no recommendations concerning therapeutic delays. Our study emphasizes that patterns of care concerning therapeutic delay did not much differ for patients less than 80 whereas it was shorter for those over 80 years of age. The reasons for this variability are numerous and the difference can be partly explained by different baseline characteristics of elderly patients and by changes in access or the physicians' treatment decisions. The reason for the increase in delays over time for patients less than 80 years is unclear as it concerned only colon cancer.

The increase may be partly related to longer access to the preoperative work-up or to a more complete assessment over time. Delays are likely to continue increasing given the continuing growth of the elderly population and the increasing use of complex, multimodal treatments (15). Neoadjuvant treatments are used for rectal cancer and require medical oncology referrals (radiation oncologists, surgeons and chemotherapists), which could lengthen the delay to the first treatment. As previously published, patients with early TNM stage I cancer had longer therapeutic delays than patients with more advanced cancers 15 . The reasons remain unclear.

Elderly patients managed in private facilities showed shorter therapeutic delays. In France, cancers are managed by a highly decentralized health care system, with many treatment facilities and many participants. The supply of care is heterogeneous and certain management disparities may be associated with the organization of the health care system or with the individual characteristics of the patients.

A recent publication has showed that the concordance between the therapies proposed during the tumor board or after the geriatric oncology consultation and the treatment actually given was satisfactory. Currently, the geriatric oncology consultation is performed quickly and does not influence the therapeutic delay (16) .

The main limit of this study is obviously its retrospective observational status, which prevented us from including relevant data, particularly on patients' 
socioeconomic level. It would have been interesting to measure how deprivation may have influenced cancer management at the population level. Information on nutrition status would also have been interesting; malnutrition is frequent and may be associated with longer hospital stays, worse outcome, impaired quality of life and performance status (17). The relatively low number of rectal cancer cases may also have led us to miss or under or overestimate some statistically relevant items.

In conclusion, this study highlights that therapeutic delays are shorter for patients over 80 years than for younger patients. Factors affecting this delay are scarce but similar in both age groups. If the range of days of therapeutic delay is not widely extended, the effect of variations in delays on patients' outcomes, such as recurrences or death, should nevertheless be explored in future research.

Conflict of interest: None

Ethical standards: The project comply with the current laws of the country.

Sponsor's Role: The sponsors of the study had no role in study design, data collection, data analysis, data interpretation, or writing of the report.

Disclaimer: None of the authors has any conflicts of interest to declare regarding this study.

Funding sources: French Institut national du cancer (INCa), Conseil Régional.

\section{References}

1. Binser-Foucard F, Belot A, Delafosse P, Remontet L, Woronoff A, Bossad N. Francim, Inserm, Institut National du Cancer. 2013.
2. Desgrippes R, Bouvier V, Delafosse P, et al. Management of rectal cancer in France in a well-defined population. Eur J Gastroen Hepat 2014;26: 743-747.

3. World Health Organisation. International Classification of Diseases (ICD). 1992.

4. Sobin L, Gospodarowicz M, Wittekind C. TNM Classification of Malignant Tumours (ed 7). Wiley, 2009, pp. 19-20.

5. Charlson ME, Pompei P, Ales KL, MacKenzie CR. A new method of classifying prognostic comorbidity in longitudinal studies: development and validation. Journal of chronic diseases 1987;40: 373-383.

6. Bouvier AM, Remontet L, Jougla E, et al. Incidence of digestive cancers in France. Gastroenterol Clin Biol 2004;28: 877-881.

7. Ramos M, Esteva M, Cabeza E, Llobera J, Ruiz A. Lack of association between diagnostic and therapeutic delay and stage of colorectal cancer. Eur J Cancer 2008;44: 510-521.

8. Pruitt SL, Harzke AJ, Davidson NO, Schootman M. Do diagnostic and treatment delays for colorectal cancer increase risk of death? Cancer causes \& control : CCC 2013;24: 961-977.

9. Singh H, De Coster C, Shu E, et al. Wait times from presentation to treatment for colorectal cancer: a population-based study. Canadian journal of gastroenterology = Journal canadien de gastroenterologie 2010;24: 33-39.

10. Neal RD. Do diagnostic delays in cancer matter? Br J Cancer 2009;101 Suppl 2: S9-S12.

11. Raje D, La Touche S, Mukhtar H, Oshowo A, Clark CI. Changing trends in the management of colorectal cancers and its impact on cancer waiting times. Colorectal Dis 2006;8: 140-144.

12. Departement of Health. Referral guidelines for suspected cancer. 2000.

13. Departement of Health. The NHS Cancer plan: a plan for investment, a plan for reform. 2000.

14. Korsgaard M, Pedersen L, Laurberg S. Delay of diagnosis and treatment of clorectal cancer--a population-based Danish study. Cancer Detect Prev 2008;32: 45-51.

15. Bilimoria KY, Ko CY, Tomlinson JS, et al. Wait times for cancer surgery in the United States: trends and predictors of delays. Ann Surg 2011;253: 779-785.

16. Blanc M, Dialla O, Manckoundia P, Arveux P, Dabakuyo S, Quipourt V. Influence of the geriatric oncology consultation on the final therapeutic decision in elderly subjects with cancer: analysis of 191 patients. The journal of nutrition, health \& aging 2014;18: 76-82.

17. Pressoir M, Desne S, Berchery D, et al. Prevalence, risk factors and clinical implications of malnutrition in French Comprehensive Cancer Centres. Br J Cancer 2010;102: 966-971. 